\title{
What is minimal about predictive inferences?
}

\author{
MARK A. MCDANIEL \\ University of New Mexico, Albuquerque, New Mexico \\ FRANZ SCHMALHOFER \\ University of Osnabrück, Osnabrück, Germany \\ and \\ DENNIS E. KEEFE \\ California State University, Fullerton, California
}

\begin{abstract}
Pronunciation of a probe word that relates to a highly predictable event is typically facilitated when the probe immediately follows the target sentence, but not when it is delayed until after a second sentence. These findings suggest that inferences about highly predictable events are minimally drawn during reading. Using a similar paradigm, in which the probe was delayed, we found significant priming in a reading condition that encouraged focus on the referential state of affairs (situation level) expressed in the sentences. In contrast, significant priming for predictive texts was not observed in a reading condition that encouraged focus on the text per se (text level). These results are interpreted within a theoretical model that assumes that predictive inferences are represented at a situational level only, whereas explicitly presented information is represented at severallevels (text and situational). It is in this sense that predictive inferences may be said to be minimally represented.
\end{abstract}

It is well established that comprehension involves both the understanding of explicitly presented information and the generation of inferred information. Furthermore, in regard to inference processes, several kinds of inferences have been identified. Some inferences are drawn to maintain referential coherence, casual coherence, or other components of textual coherence. In contrast, there are a host of inferences that are not required to maintain textual coherence, inferences that have been termed elaborative. Elaborative inferences could supply a typical object for a verb, such as inferring hammer for pounding a nail (instrumental inferences). Elaborative inferences could add appropriate shades of meaning to a concept, such as inferring round for tomato when reading the girl rolled a tomato (semantic inference). They could produce information about the likely outcome of an event, such as inferring that an actress died, upon reading the director and the cameraman were preparing to shoot closeups of the actress on the edge of the roof of the 14th story building when suddenly the actress fell. This last type of elaborative inference has been termed a predictive inference, and it is this type of inference that is the focus of the present study.

A fundamental theoretical debate has centered on the extent to which predictive inferences are routinely generated during comprehension. There have been several theo-

Correspondence concerning this article should be addressed to M. A. McDaniel, Department of Psychology, Logan Hall, Room 108, University of New Mexico, Albuquerque, NM 87131-1161(e-mail: mcdaniel@ unm.edu). retical positions that have been entertained. One is that predictive inferences are not generated by readers. This view is supported by Potts, Keenan, and Golding's experiments (1988, Experiments 3 and 4), in which subjects read a target sentence (as above) followed by a second sentence (The director was talking to the cameraman and did not see what happened). Then, the subjects were required to pronounce a probe word ("dead") as quickly a possible. The latency to pronounce the probe word was not facilitated by the related target sentence (relative to a target sentence that could not support the inference). In contrast, when the second sentence (Her orphaned daughters sued ...") required the inference to maintain causal coherence (a bridging inference condition), the latency to pronounce the probe was reliably faster than that for the control.

Keefe and McDaniel (1993) extended the Potts et al. (1988) study, finding that if the probe was presented immediately after the first sentence, there was evidence for the predictive inferences (see also Murray, Klin, \& Myers, 1993, Experiment 2). As in Potts et al., if the probe was delayed until after the second sentence was presented, there was no longer any evidence that the inference had been activated. This is in contrast to the intact priming after the second clause that was observed when the proposition that the actress had died was explicitly stated in the target sentence. These data suggest that forward inferences are drawn but that the dynamics of their representation and maintenance do not parallel that of textually presented information. One existing view that may be applied to these findings is that predictive inferences are generated, but only in a tenuous or minimal fashion (McKoon \& Ratcliff, 
1986). The basic idea of the minimalist position is that the inference is not fully drawn (died, in the example above) but, instead, contains only a few of the semantic features associated with the complete inference (e.g., that something bad happened to the actress). Presumably, this putative minimal encoding is also more fragile, because the data suggest that it is present only momentarily (Keefe \& McDaniel, 1993).

Another theoretical framing of the predictive-inference findings is stimulated by research concerning the representation of discourse in memory (for a review, see Fletcher, 1994). Briefly, van Dijk and Kintsch (1983) have proposed that the comprehension of a text entails more than merely constructing a representation of the text per se; it also includes the construction of a mental representation of the state of affairs described by the sentences of the text (termed the situation level). This proposal has stimulated much empirical work identifying differences between text and situational-levelrepresentations. Text-level representations reflect the linguistic structure of the text and the meaning of the text elements (e.g., propositions). Situational representations are mental models that are, to a certain degree, independent of the text, in that a situation model may also result from perception or problem solving, rather than solely from comprehending a text. In text comprehension, a situation model will usually represent the referential state of affairs that is addressed by the text (the situation conveyed by the text). Text and situational levels have been experimentally distinguished, most prominently through recognition tasks that probe memory with several types of foils designed to index each representational level (see Fletcher, 1994, for amplification).

Furthermore, this recognition procedure has been used to show that instructions that focused the reader's attention toward the situation yielded a stronger situational representation and a weaker text representation, whereas instructions that focused the reader's attention toward the text yielded stronger text representations and a weaker situational representation (see Schmalhofer \& Glavanov, 1986, Figure 3). Thus, it has been established that different reading instructions may focus the reader's attention toward the situational level, on the one hand, and toward the text level, on the other hand (see Barclay, 1973, for converging evidence). In the present study, we use this instructional technique to investigate the following claim. We suggest that predictive inferences are represented at the situation level, whereas explicitly presented textual information is represented not only at the situational level, but at the text level as well. It is in this sense that the predictive inference may be said to be tenuous or minimal, because it is not also represented at a text (propositional) level, as are explicit statements and other types of inferences (e.g., see Keenan \& Kintsch, 1974).

The details of this theoretical view are provided by Schmalhofer, McDaniel, and Keefe (in press); here, we can only highlight the pertinent aspects. The view adopts Kintsch's (1998) construction-integration theory, which proposes that the reader generally constructs an intercon- nected multilevel representation of the text (this assumption is supported by the research on discourse representation mentioned above). At each level, information is represented by nodes that are linked together within and across levels to form a network structure that is the cognitive representation of the comprehended text. Furthermore, the nodes carry variable activation levels, with the activation level reflecting the degree to which the nodes fit with the emerging network structure. High activation levels are associated with nodes that fit well with the network structure, and low activation levels indicate nodes that are disconnected from the network. Briefly, we suggest that after a target clause (e.g., when suddenly the actress fell), an inference is constructed from world knowledge (in this case, about what can happen when falling from high heights). The situation node representing that inference is highly activated, because it is strongly interconnected with other situation nodes (e.g., reflecting other inferences about falling). Thus, priming is produced if pronunciation to a probe is tested immediately (the activation levels of the situation node representing the inference are assumed to contribute to the priming effects in the word-pronunciation tasks used in experimental studies). Importantly, however, when a second sentence is read that is unrelated to the predictive inference, the critical situation node is not very well interconnected to the emerging network. At this point, the activation level is low, and priming is not produced.

In contrast, in an explicit condition (e.g., ... when suddenly the actress fell and was pronounced dead), the target information (dead) has been represented at the text level, as well as at the situational level. Being recently presented, this information is carried over to influence the integration of the subsequent sentence (The director was talking to the cameraman and did not see what happened). Accordingly, high activation of the target information at the situational level remains, and priming is produced for the explicit condition. Finally, if the second sentence connects well with the critical situation node (Her orphaned daughters sued the director and the studiofor negligence), that node stays well connected to the network, and priming is produced (this is the bridging inference condition).

The present experiment was conducted to provide an initial investigation of our core idea that the persistence of predictive inferences rests on activation at the situational level, rather than representation at the text (propositional) level (cf. Keenan \& Kintsch, 1974; Kintsch, 1988). If this evidence were forthcoming, the theoretical notion of a minimal inference could be more precisely instantiated. As was mentioned earlier, our experimental strategy was to manipulate reading instructions so that subjects had to focus either on the text or on the situation described by the text. If priming is due to representations or activation at the situational level, the priming effect should get stronger when a reader focuses on the construction of the situation model during reading. On the other hand, if activation (or more specifically, interconnectivity) at the text level is responsible for the priming effect, focusing on the text 
proper during reading ought to enhance the priming effect. We used the two different types of reading instructions established by Barclay (1973) and Schmalhofer and Glavanov (1986) to stimulate activation of either situationallevel representations or text-level representations.

On the basis of our theoretical approach, our primary prediction was that when the reading focus is on the situational level, a robust priming effect will occur in the predictive inferencing condition, so that priming will be obtained even after the presentation of a second sentence in the predictive inferencing condition. This is because the situation-focused reading instruction yields a more elaborate situationalelaboration, so that previously unconnected situational nodes are now connected, thereby conferring activation to the inference (situation) node. In contrast, in the text-focused reading, no such elaborations are stimulated, and no priming in the predictive inference condition is predicted. It is worth noting that the prediction for priming in the situation-focused reading group is a strong one. No study to date has shown priming when the target sentence is followed by a second sentence that does not support the inference suggested by the target sentence (except when sentences are presented in fragmented form; Keefe \& McDaniel, 1993).

Further converging on the model is the expectation that the probe latency for the explicit condition, if influenced at all by the situation-focused reading instructions, might become slower. For the explicit condition, we assume that priming is supported by interconnectivity (activation) at the propositional level. Situation-focused reading instructions should divert activation to the situational level, thereby reducing the activation at the propositional level. Thus, our model raises the possibility that the response speed (and the priming) for the explicit condition will slightly decrease in the situation-focused reading condition relative to the text-focused reading condition (this prediction emerges from simulations of the model reported in Schmalhofer et al., in press).

There was one other feature of the experiment that merits comment. Using recognition tests, we attempted to independently establish that the situation-focused reading instructions encouraged subjects to focus on construction of a situational representation more so than did the textfocused reading instructions. Specifically, we required subjects to make recognition judgments for probes that were explicitly presented in the text, probes that reflected the critical target inference in the predictive and bridging conditions (explicitly presented in the explicit condition), probes that reflected an elaboration of that target inference (or an elaboration of the explicit statement in the explicit condition), and probes that were inconsistent with the text (and any possible inferences). We reasoned that greater focus on extracting a situational representation of the text should produce more endorsements for inference and elaboration probes (relative to the text-focused condition). Importantly, the anticipated higher endorsement rate for the situation-focused condition should not extend to the explicit texts for the inference probes. The explicit texts directly state the information in the inference probe, so that the text-focused condition would focus readers on the information as well as did the situation-focused condition. In contrast, the explicit texts do not state the information of the elaboration probe. The elaboration information must be inferred in the explicit texts, and accordingly situation-focused reading ought to increase the likelihood that such information is captured in the representation of the text. Thus, situation-focusedreading should produce higher endorsement rates than does text-focused reading for elaboration probes, but for not for inference probes of explicit texts. Finally, endorsement rates for inference and elaboration probes should not be higher in the situationfocused condition (relative to the text-focused condition) for the control texts, since these texts do not support the inference or its related elaboration.

\section{METHOD}

\section{Design and Subjects}

The experiment was a 2 (instructions) $\times 4$ (text) mixed factorial design. Type of instructions was manipulated between subjects and consisted of situation-focused reading instructions or text-focused instructions. Text type was manipulated within-subjects and included predictive, bridging, explicit, and control passages. Ninetysix undergraduates from California State University, Fullerton, participated in the experiment for course credit. Forty-eight subjects were randomly assigned to each of the two instructional conditions.

\section{Materials}

Passages were obtained from previous research (Keefe \& McDaniel, 1993; Potts et al., 1988). The 40 passages were revised to eliminate argument overlap across passages. The passages consisted of two sentences each. The four different passages contained the same content words but differed in implication (see Table 1). Test items included single words for the pronunciation task and sentences for the recognition task. Pronunciation test items included inferencerelated words (e.g, dead) and unrelated items. Inference targets were included in only half of the trials, in order to reduce the predictability of the pronunciation target. Thus, there were five inference-related targets presented to each subject within each text passage condition.

In the recognition task, there were four types of recognition probes (see Table 1), and these probes were used for all of the passage types. The explicit probe was taken almost verbatim from the original passage. Inference probes included the inference in fullsentence form. Note that the inference probes reflected explicitly stated information from the explicit passages and thus, for those passages, the "inference" alternative would be a correct response. Elaboration probes provided more detail regarding the inferred event. Inconsistent probes directly contradicted the inference. To reduce predictability, only two probes were presented for testing after any particular passage. This resulted in each subject's receiving five recognition probes of each type (explicit, inference, elaboration, and inconsistent) for every type of passage.

\section{Procedure}

The subjects were first told that the study involved comprehension and that they would be tested on the material after having read it. In the situation condition, they were told to elaborate on the context described in the passage, imagining the details of the situation, those involved, and their actions. In the text condition, the subjects were told to concentrate on the precise wording of the sentences and not to concern themselves with anything else.

On each trial, the subjects were shown the two-sentence passage, a pronunciation target, and then two recognition test probes. During 
Table 1

Sample Material for the Text Passages and of the Four Different Recognition Probes

Text Passages

Predictive

The director and the cameraman were preparing to shoot closeups of the actress on the edge of the roof of the 14th story building when suddenly the actress fell. The director was talking to the cameraman and did not see what happened.

\section{Bridging}

The director and the cameraman were preparing to shoot closeups of the actress on the edge of the roof of the 14th story building when suddenly the actress fell. Her orphaned daughters sued the director and the studio for negligence.

Explicit

The director and the cameraman were preparing to shoot closeups of the actress on the edge of the roof of the 14th story building when suddenly the actress fell and was pronounced dead. The director was talking to the cameraman and did not see what happened.

Control

Suddenly, the director fell over the camera stand, interfering with the cameraman's efforts to shoot closeups of the actress on the 14th

floor. By the time the camera equipment was set up again, it was too dark to continue shooting.

\section{Recognition Alternatives}

Explicit probe

The cameraman was preparing to shoot closeups.

Inference probe

The actress was pronounced dead.

Elaboration probe

The actress died from her injuries.

Inconsistent probe

The actress lived a long life.

reading, sentences were presented on the computer screen one at a time. The subjects pressed a key to receive each sentence. Following the passage, a row of asterisks appeared for $250 \mathrm{msec}$, after which a single word target was presented for $1 \mathrm{sec}$. The subjects were asked to pronounce the word aloud as quickly as possible. One second after the target was shown, the first recognition probe was presented for $3 \mathrm{sec}$. The subjects were told to press the Enter key if the statement was explicitly mentioned in one of the sentences of the passage and to press the space bar if not. They were also instructed to respond quickly without making too many errors. Three seconds after onset of the first probe, the second recognition probe was presented. There were 40 such trials.

In the pronunciation task, reaction times (RTs) were measured from the onset of the target to the initiation of the vocal response. These responses were measured using a microphone that was connected to a Lafayette Instruments Voice Activated Relay, which was connected to a personal computer.

\section{RESULTS}

\section{Pronunciation Latencies}

For all statistical tests, the rejection level for statistical significance was set at .05. Table 2 shows the RTs for the pronunciation task of the different experimental conditions. The data were initially analyzed in a 2 (instructions) $\times 4$ (text passage) mixed factorial analysis of variance (ANOVA).

There was a significant difference among the four text versions [predictive, bridging, explicit and control; $F(3,282)=$ 9.19, $\left.M S_{\mathrm{e}}=5,599.15\right]$, with explicit texts producing the fastest responses and control texts the slowest responses. More pertinent for present purposes, there was a significant interaction between text version and reading instructions $\left[F(3,282)=2.04, M S_{\mathrm{e}}=5,599.15\right]$. To interpret this interaction, priming effects were calculated for each reading instruction group as the difference between the control text passage condition and each of the other text passage conditions. Following Loftus and Masson (1994), confidence intervals were calculated for the differences between these means (CIMD $=30.2$ ). In line with previous findings, in the text-focused reading group, significant priming was observed in the explicit and bridging conditions, but not in the predictive condition. In contrast, in the situation-focused reading group, as was expected, significant priming was observed also for the predictive condition.

Finally, a $2 \times 2$ mixed ANOVA limited to the predictive and explicit text conditions revealed a significant interaction between text version and reading instructions $[F(1,94)=$ $\left.4.89, M S_{\mathrm{e}}=5,273.55\right]$. Examination of Table 2 shows that the interaction confirmed the theoretical predictions. Response latencies in the predictive condition decreased with situation-focused reading relative to text-focused reading, whereas in the explicitcondition situation-focused reading increased latencies relative to text-focused reading. This pattern also indicates that situation-focused reading does not produce a generalized reduction in response latencies to the inference probe $(F<1$ for the reading instruction main effect). This ANOVA also found a general advantage in probe latency for the explicit condition relative to the predictive condition $\left[F(1,94)=4.33, M S_{\mathrm{e}}=5,273.55\right]$.

\section{Recognition Responses}

Table 3 shows the average frequencies of yes responses to the inference probes, elaboration probes, explicit probes, and inconsistent probes as a function of text condition (predictive, bridging, explicit, and control) and two reading instructions (text- or situation-focused reading). Separate 4 (text passage) $\times 2$ (instructions) mixed ANOVAs on the proportion of yes responses for the inference, elaboration, explicit, and inconsistent recognition probes were conducted.

For the inference probes, there was no significanteffect of reading instructions $(F<1)$. As was predicted, however, there was a significant interaction between reading in-

Table 2

Reaction Times (in Milliseconds) in Pronunciation Task (to Inference Targets)

\begin{tabular}{lcccc}
\hline Condition & Predictive & Bridging & Explicit & Control \\
\hline Situation & 569 & 556 & 571 & 614 \\
Text & 595 & 576 & 550 & 609 \\
\hline
\end{tabular}

Note-Error rates were virtually zero. 
Table 3

Relative Frequencies of "Yes" Responses to the Four

Recognition Probes for the Four Text Types Under the Two Reading Instructions

\begin{tabular}{|c|c|c|c|c|c|c|c|c|}
\hline \multirow[b]{3}{*}{ Recognition Probe } & \multicolumn{8}{|c|}{ Text Types } \\
\hline & \multicolumn{2}{|c|}{ Predictive } & \multicolumn{2}{|c|}{ Bridging } & \multicolumn{2}{|c|}{ Explicit } & \multicolumn{2}{|c|}{ Control } \\
\hline & $\begin{array}{c}\text { Text } \\
\text { Focused }\end{array}$ & $\begin{array}{l}\text { Situation } \\
\text { Focused }\end{array}$ & $\begin{array}{c}\text { Text } \\
\text { Focused }\end{array}$ & $\begin{array}{l}\text { Situation } \\
\text { Focused }\end{array}$ & $\begin{array}{c}\text { Text } \\
\text { Focused }\end{array}$ & $\begin{array}{c}\text { Situation } \\
\text { Focused }\end{array}$ & $\begin{array}{c}\text { Text } \\
\text { Focused }\end{array}$ & $\begin{array}{l}\text { Situation } \\
\text { Focused }\end{array}$ \\
\hline Explicit & .72 & .82 & .75 & .94 & .77 & .86 & .51 & .52 \\
\hline Inference & .22 & .29 & .27 & .36 & .93 & .87 & .15 & .11 \\
\hline Elaboration & .24 & .33 & .26 & .42 & .23 & .38 & .15 & .16 \\
\hline Inconsistent & .11 & .12 & .14 & .05 & .11 & .04 & .12 & .11 \\
\hline
\end{tabular}

structions and text type such that situation-focused instructions produced a higher endorsement rate for predictive and bridging texts, but not for explicit and control texts $\left[F(3,282)=3.90, M S_{\mathrm{e}}=0.87\right]$. There was also a significant main effect of text type $\left[F(3,282)=323.26, M S_{\mathrm{e}}=\right.$ $0.87]$. Inspection of Table 3 confirms that this effect occurs because the subjects appropriately rejected the inference probe after control texts (for which the inference was not supported) and endorsed the probe at a high rate after explicit texts (for which the "inference" was explicitly stated). Endorsement rates for the predictive and bridging texts were between these extremes.

For the elaboration probes, the situation-focused instructions produced significantly more yes responses than did the text-focused instructions $\left[F(1,94)=16.06, M S_{\mathrm{e}}=\right.$ 1.53]. As was expected, however, Table 3 shows that the higher endorsement rate for the situation-focused instructions was not present for the control texts. This observation was verified by a significant interaction between reading instructions and text type $\left[F(3,282)=3.02, M S_{\mathrm{e}}=\right.$ 0.99]. The main effect of text type was also significant $\left[F(3,282)=15.11, M S_{\mathrm{e}}=0.99\right]$, indicating that for the control texts, the subjects were less likely to endorse the elaboration recognition probe than for the other texts. Thus, as was outlined in the introduction, the endorsement patterns for inference and elaboration probes are consistent with the assumption that situation-focused reading instructions did produce more focus on a situational representation than did text-focused reading instructions.

For the explicit text probes, endorsement rates were relatively high, with situation-focused reading producing a greater proportion of yes responses than did text-focused reading $\left[F(1,94)=20.17, M S_{\mathrm{e}}=1.14\right]$. This effect did not emerge for control texts, however $\left[F(3,282)=15.11, M S_{\mathrm{e}}=\right.$ 1.19 , for the interaction]. The main effect of text type was significant as well $\left[F(3,282)=46.12, M S_{\mathrm{e}}=1.19\right]$, with control texts showing lower performance (less accurate) than did other texts. The explicit probes differed in content for the control relative to the other texts, and this effect may indicate that the probes differed in recognition difficulty.

For the inconsistent probes, endorsement rates were generally low, indicating accurate rejection of incorrect statements. The ANOVA on the proportion of yes responses revealed that situation-focused reading produced significantly more accurate responding (lower proportion of yeses) than did text-focused reading $[F(1,94)=6.23$, $M S \mathrm{e}=0.64]$, but this pattern held only for the bridging and explicit texts $\left[F(3,282)=3.80, M S_{\mathrm{e}}=0.37\right.$, for the interaction; see Table 3].

The strength of a situational representation can be assessed by scoring the endorsement responses to inference statements as hits and the endorsement responses to inconsistent statements as false alarms (see Kintsch, 1998, pp. 251-253; Schmalhofer \& Glavanov, 1986) in a signal detection analysis. From these hits and false alarm rates, a $d^{\prime}$ value (Green \& Swets, 1966) is then calculated, which assesses the relative strength of the situational representation. Such an analysis was performed with the endorsement rates to the elaboration statements (hits) (because these statements reflect inferential processes for the explicit text conditions, as well as for the predictive and bridging conditions) and to the inconsistent statements (false alarms) under the two reading instructions. ${ }^{1}$

In the text-focused reading condition, the measured strengths of the situational representation were $d^{\prime}=0.54$ (predictive), $d^{\prime}=0.48$ (bridging), and $d^{\prime}=0.53$ (explicit text condition). In the situation-focused reading condition, the strengths of the situational representation were $d^{\prime}=$ 0.80 (predictive), $d^{\prime}=1.48$ (bridging), and $d^{\prime}=1.39$ (explicit text condition). A $2 \times 3$ mixed ANOVA for these data confirmed that the $d^{\prime}$ scores were significantly higher in the situation-focused reading condition than in the textfocused reading condition $\left[F(1,94)=22.50, M S_{\mathrm{e}}=1.62\right]$. There was also a significant main effect of text type $\left[F(2,188)=3.31, M S_{\mathrm{e}}=0.88\right]$, and a significant interaction of text type and reading condition $[F(2,188)=4.23$, $\left.M S_{\mathrm{e}}=0.88\right]$. This method of analyzing the recognition data converges on the assumption that the situational representation was uniformly stronger in the situation-focused reading condition than in the text-focused reading condition.

\section{DISCUSSION}

In the situation-focused reading condition, significant priming for the probe word was observed, implying activation of a forward (predictive) inference. This is an important empirical result with these materials, because it extends the conditions under which predictive inferences appear to be active. Typically, when the probe is presented after the second sentence, a sentence that does not fore- 
ground the possible consequences of the first sentence, there is no evidence for the presence of a predictive inference (i.e., priming for the probe; Keefe \& McDaniel, 1993, Experiments 2 and 3; Potts et al., 1988).

Perhaps of more consequence, the result is theoretically informative. Our theoretical position suggests that predictive inferences are drawn but initially are represented only at a situational level. This theory explicitly predicts (Schmalhofer et al., in press, provide the formal simulations) that significant priming for the probe should be obtained (with the predictive texts) even when the probe is delayed until after a second sentence, provided that the situational representation continues to receive activation. Furthermore, the theory predicts that priming for the probe would not be observed (when the probe is presented after the second sentence) in the predictive inference conditions when the situational representation was not a specific focus during reading. The priming in the situationfocused condition, coupled with the absence of priming in the text-focused condition, is entirely consistent with these predictions. The absence of priming in the text-focused condition converges on identical findings repeatedly reported under standard reading conditions (Keefe \& McDaniel, 1993; Potts et al., 1988).

The probe latency patterns in the explicit condition further support the interpretation that predictive inferences are represented at the situational level. By all accounts, explicitly presented information would be primarily maintained by activity at the two text levels (propositional and, perhaps, verbatim levels). This kind of representation presumably would not be activated by a situational focus; as was found in this experiment, a situation-focused condition produced a nominal increase in probe latency for explicit statements (relative to the text-focused condition). This pattern was the reverse of that found for the inference conditions.

The recognition data provide supportive evidence that situation-focused instructions did focus readers on the situational representation, more so than did text-focused instructions. Readers given situation-focused instructions were more likely than readers given text-focused instructions to remember the texts as containing inferences and elaborations that were not explicitly stated. This finding demonstrates that situation-focused reading instructions stimulated processing that went beyond that produced by focus on the text elements, and such additional processing is captured in the present model by activation at the situational level of the text representation.

Some theorists (e.g., Forster, 1981) have cautioned that on-line tests of inferencing do not necessarily implicate inference-based priming of the test probe but, rather, interactions between the meaning of the sentence in shortterm memory and the test item (which is simultaneously in short-term memory). This interpretation does not appear plausible for the present results, for several reasons. First, in the present paradigm, the critical target sentence would likely not be in short-term memory, having been replaced by the second sentence that intervened between the target sentence and the test item. Second, even if the target sentence were still residing in short-term memory, on this account both reading instructions should have produced priming for the predictive condition.

One may still possibly worry that the experimental effects from the manipulation of reading instructions might stem from situation-focused reading's yielding longer reading times than did text-focused reading. Longer reading times in the situation-focused group might produce a more activated short-term memory representation (thereby perhaps supporting a possible noninference-based priming process, as was just mentioned), or more time might simply allow more inferencing. Existing research, however, indicates that the difference in reading times is the other way around. In an experiment in which reading times were collected with the same text materials (Vogel, 1997), the text-focused readers read for significantly longer than the situation-focused readers. This same result was obtained in another experiment with similar kinds of textand situation-focused reading instructions, (see Schmalhofer \& Glavanov, 1986, Figure 2). Therefore, the experimental effects are probably not explained by reading time differences but, instead, by different memory strengths in the text versus situation encodings.

In contrast to the present theoretical approach, some frameworks for forward inferencing would not necessarily predict the present finding that forward inferences remained active after presentation of the second sentence only in the condition wherein the situational representation received focal attention (with materials of the type used here). One general view posits that once inferences are drawn, they are represented at a propositional level (Keenan \& Kintsch, 1974). Given that the predictive inferences appear to be drawn for the materials present (Keefe \& McDaniel, 1993), the implication is that activation of the inference ought to persist (to the extent that activation of the explicitly presented information persists). This expectation clearly is not consistent with the present results in the text-focused condition and most past findings.

The present theoretical framework provides an advance over several current views as well by suggesting a more precise formulation of the nature of forward inferences. One current view suggests that forward inferences are minimally drawn and are only marginally represented (or activated) unless subsequent text reinforces the inference (McKoon \& Ratcliff, 1992; Singer, Halldorson, Lear, \& Andrusiak, 1992). One drawback to this view, as currently developed, is that the general idea of a minimally drawn inference is not firmly specified (e.g., in terms of content, activation, or representation). Accordingly, the theoretical notion has limited predictive power. Still, in post hoc fashion, this notion might account for the present findings by suggesting that forward inferences are fully drawn under conditions in which the reader is focusing primarily on the situational information conveyed by the text and are, accordingly, maintained subsequent to the target (first) sen- 
tence. However, this explanation does not incorporate the additional finding of a reverse pattern in latencies for the explicit condition.

Another current view, the constructionist view of text comprehension (Graesser, Singer, \& Trabasso, 1994), holds that predictive inferences may be generated in a supportive context. Perhaps the situation-focused reading instructions might be construed by these theorists as being a supportive context. Note, however, that this view more prominently suggests that "readers do not normally construct inferences that forecast future episodes in the plot" (Graesser et al., 1994, p. 372). On our model, the inference is typically drawn at the situational level, even under conditions that do not specifically focus the reader on situational aspects of the text. Because the inference is represented at the situational level, it will be reflected in the knowledge network (even though it will not necessarily remain active unless further text reinforces the inference or unless the reader, for whatever reason, focuses on the situational representational level). Thus, if later text inputs afford a boost in activation for that situational node, the inference will be evidenced. Consistent with this idea, Klin, Murray, Levine, and Guzman (1999) have also found that forward inferences are more prevalent and more persistent than has previously been assessed.

More specifically, McKoon and Ratcliff (1986) found that even after intervening unrelated texts, a recognition probe (e.g., dead) was more difficult to reject with the predictive texts (relative to control texts), but only when a prime (the main character of the passage) preceded the recognition probe (Experiment 4). This result has been taken as evidence for the persistence of the inference representation. Assuming that these recognition data do implicate inference persistence, ${ }^{2}$ our framework suggests that evidence for the inference was manifested because presentation of the main character of the passage would boost activation of the situational-level representation. In contrast to this explanation, the minimalist interpretation of these results is that the prime may change the nature of the information represented by the inference. For example, the prime might add specificity to the minimal encoding "something bad" or might override conjoining information encoded with the inference that it was "not explicitly stated" (McKoon \& Ratcliff, 1986, p. 88). This interpretation is challenged by the question of how it is that presenting the main character of the passage as a prime would now produce a more specific or explicit representation of a particular minimally drawn inference when the full predictive sentence did not do so. Clearly, however, further experimentation is needed to explore the contrast among the minimalist, the constructionist, and the present accounts. At this point, one might favor the situational account, because it explicitly anticipated the present effects of reading conditions on the presence of priming in the predictive inference condition.

\section{REFERENCES}

BARCLAY, J. R. (1973). The role of comprehension in remembering sentences. Cognitive Psychology, 4, 229-254.

Fletcher, C. R. (1994). Levels of representation in memory for discourse. In M. A. Gernsbacher (Ed.), Handbook of psycholinguistics (pp. 589-607). San Diego: Academic Press.

ForSTER, K. (1981). Priming and the effects of sentence and lexical contexts on naming time: Evidence for autonomous lexical processing. Quarterly Journal of Experimental Psychology, 33A, 465-495.

Graesser, A. C., Singer, M., \& Trabasso, T. (1994). Constructing inferences during narrative text comprehension. Psychological Review, 101, 371-395.

Green, D. M., \& Swets, J. A. (1966). Signal detection theory and psychophysics. New York: Wiley.

KeEfe, D. E., \& McDAniel, M. A. (1993). The time course and durability of predictive inferences. Journal of Memory \& Language, 32, 446-463.

KeEnan, J., \& KintsCH, W. (1974). The identification of explicitly and implicitly presented information. In W. Kintsch (Ed.), The representation of meaning in memory (pp. 153-165). Hillsdale, NJ: Erlbaum.

KINTSCH, W. (1988). The role of knowledge in discourse comprehension: A construction-integration model. Psychology Review, 95, 163-182.

Kintsch, W. (1998). Comprehension: A paradigm for cognition. New York: Cambridge University Press.

Klin, C. M., Murray, J. D., Levine, W. H., \& Guzman, A. E. (1999). Forward inferences: From activation to long-term memory. Discourse Processes, 27, 241-260.

Loftus, G. R., \& Masson, M. E. J. (1994). Using confidence intervals in within-subject designs. Psychonomic Bulletin \& Review, 1, 476-490.

McKoon, G., \& Ratcliff, R. (1986). Inferences about predictable events. Journal of Experimental Psychology: Learning, Memory, \& Cognition, 12, 82-91.

McKoon, G., \& Ratcliff, R. (1992). Inference during reading. Psychological Review, 99, 440-466.

Murray, J. D., Klin, C. M., \& Myers, J. L. (1993). Forward inferences in narrative text. Journal of Memory \& Language, 32, 464-473.

Potts, G. R., Keenan, J. M., \& Golding, J. M. (1988). Assessing the occurrence of elaborative inference: Lexical decision versus naming. Journal of Memory \& Language, 27, 399-415.

Schmalhofer, F., \& Glavanov, D. (1986). Three components of understanding a programmer's manual: Verbatim, propositional, and situational representations. Journal of Memory \& Language, 25, 279-294.

Schmalhofer, F., McDaniel, M. A., \& Keefe, D. (in press). A unified model for predictive and bridging inferences. Discourse Processes.

Singer, M., Halldorson, M., Lear, J. C., \& Andrusiak, P. (1992). Validation of causal bridging inferences. Journal of Memory \& Language, 31, 507-524.

VAN DiJK, T. A., \& KINTsch, W. (1983). Strategies of discourse comprehension. San Diego: Academic Press.

Vogel, R. (1997) Inferenzen beim wortlaut- und ereignisfokusierten Lesen von Texten [Inferences in the word-and event-focused reading of texts]. Diplomarbeit (master's thesis), University of Mannheim, Department of Psychology.

\section{NOTES}

1. In each text condition, from $54 \%$ to $60 \%$ of the subjects had either zero false alarms or $100 \%$ hits. To calculate $d^{\prime}, .02$ was substituted for a $0 \%$ false alarm rate and .98 was substituted for a $100 \%$ hit rate.

2 . Some theorists have argued that the interpretation of such recognition data is ambiguous because memory processes operative at the time of recognition, rather than inferences drawn at the time of reading, may be responsible for the effects (e.g., Potts et al., 1988).

(Manuscript received May 2, 2000; revision accepted for publication February 1, 2001.) 